\title{
Synchronizing Models and Code (Invited Talk)
}

\author{
Oscar Nierstrasz \\ Software Composition Group \\ University of Bern, Switzerland \\ http://scg.unibe.ch
}

\begin{abstract}
Object-oriented development promotes the view that "programming is modeling". Nevertheless, it remains difficult to correlate domain concepts and features with source code, to reconcile static and dynamic views of object-oriented code, and to evolve software of a running system. There continues to be a significant gap between high-level models of software applications and the code that realizes these models. We review some recent research of the Software Composition Group that attempts to address these shortcomings, and we put forward some challenges for future object-oriented development systems.
\end{abstract}

